Revta brasil. Bot., São Paulo, V.23, n.2, p.207-215, jun. 2000

\title{
Estrutura genética espacial em populações naturais de Machaerium villosum Vog. (Leguminosae) na região de Moji-Guaçu, SP, Brasil ${ }^{1}$
}

\author{
JOÃO DEL GIUDICE NETO ${ }^{2,4}$ e PAULO YOSHIO KAGEYAMA ${ }^{3}$
}

(recebido em 23 de junho de 1999; aceito em 28 de fevereiro de 2000)

\begin{abstract}
Spatial genetic structure within natural populations of Machaerium villosum Vog. (Leguminosae) at Moji-Guaçu region, SP, Brazil). A spatial autocorrelation study of enzyme loci detected by starch gel electrophoresis was performed to verify the occurrence of spatial genetic structure within two natural populations of Machaerium villosum Vog. The sampled populations were termed "Antropic Model (MA)" and "Natural Model (MN)" and they are situated in Campininha Farm areas, at Moji-Guaçu municipality, $22^{\circ} 10^{\prime} 43^{\prime}$ "$22^{\circ} 18^{\prime} 19^{\prime \prime}$ S and $47^{\circ} 8^{\prime} 5^{\prime \prime}-47^{\circ} 11^{\prime} 34^{\prime \prime} \mathrm{W}$, in the state of São Paulo. Ten polymorphic loci in the MA population and nine polymorphic loci in the MN population were assessed by Moran's I autocorrelation statistic. No spatial autocorrelation was detected among individuals within sampled populations. Results are in line with other studies in woody species from tropical rain forest.
\end{abstract}

RESUMO - (Estrutura genética espacial em populações naturais de Machaerium villosum Vog. (Leguminosae) na região de Moji-Guaçu, SP, Brasil). Foi realizado um estudo de autocorrelação espacial em locos enzimáticos determinados por meio de eletroforese em gel de amido, com o objetivo de verificar a existência de estrutura genética espacial entre os indivíduos de duas populações naturais de Machaerium villosum Vog. As populações amostradas foram denominadas "Modelo Antrópico (MA)" e "Modelo Natural (MN)",

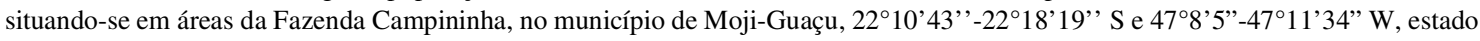
de São Paulo. Dez locos polimórficos na população MA e nove na população MN foram analisados por meio da estatística I de Moran de autocorrelação espacial. Não foi detectada a existência de estruturação genética entre os indivíduos dentro das populações estudadas. Os resultados são similares aos obtidos em outros estudos com espécies arbóreas da floresta tropical.

Key words - Machaerium villosum, allozymes, spatial autocorrelation

\section{Introdução}

A distribuição espacial da variabilidade genética dentro de populações naturais de plantas pode influenciar significativamente os processos evolutivos e ecológicos dessas populações, resultando em agregados de genótipos específicos (Shapcott 1995). Dentre os fatores que podem levar à formação de estrutura espacial estão a diferenciação de um caráter, em resposta a um gradiente ambiental, a heterogeneidade entre ambientes, associada à homogeneidade dentro destes ambientes, fatores históricos, como a extinção local e a recolonização por migrantes, e a dispersão limitada de pólen e sementes, a qual favorece cruzamentos entre indivíduos próximos, causando o isolamento pela distância (Sokal \& Oden 1978b, Epperson 1995, Loiselle et al. 1995).

1. Parte da dissertação de mestrado de J.D. Giudice Neto.

2. Seção de Ecologia, Instituto de Botânica, Caixa Postal 4005, 01061-970 São Paulo, SP, Brasil.

3. Departamento de Ciências Florestais, ESALQ/USP, Caixa Postal 9, 13418-900 Piracicaba, SP, Brasil.

4. Autor para correspondência: jdgiudic@zipmail.com.br.
A seleção em microhabitats é particularmente importante quando os ambientes variam pronunciadamente. Por outro lado, os processos genéticos que fundamentam os padrões de variação espacial observados em frequiências alélicas são mutação, seleção, migração e isolamento pela distância (Sokal et al. 1989, Sokal \& Jacquez 1991).

Os estudos sobre a estrutura espacial de populações são importantes, pois a estruturação afeta a estimativa de muitos parâmetros genéticos populacionais, como por exemplo a taxa de cruzamento (Epperson 1989, Epperson \& Allard 1989). O conhecimento sobre a estrutura espacial populacional é importante, ainda, quando populações de plantas são selecionadas para conservação ou coletadas para uso em programas de melhoramento (Shapcott 1995). Isto deve ser considerado a fim de estabelecerem-se estratégias de amostragem de populações naturais, conseguindo-se assim, segundo Epperson (1989), maximizar a diversidade populacional ou da espécie. A aplicação deste conhecimento também tem o efeito de minimizar o impacto de práticas de manejo sustentáveis dos recursos genéticos. Ainda, o estudo dos padrões espaciais sobre a variabilidade genética podem levar a novas introspecções sobre as causas que determinam a partilha observada entre a variação 
entre e dentro das populações (Leonardi \& Menozzi 1996).

Intuitivamente, suspeita-se a existência de estruturação dentro das populações naturais, contudo, a detecção de uma pronunciada estrutura tem frustrado os geneticistas, sobretudo os de espécies arbóreas (Perry \& Knowles 1991).

A introdução das técnicas de autocorrelação espacial em estudos biológicos (Sokal \& Oden 1978a,b) estimulou o surgimento de uma série de investigações sobre as populações arbóreas (Dewey \& Heywood 1988, Epperson \& Allard 1989, Perry \& Knowles 1991, Shapcott 1995, Leonardi \& Menozzi 1996). A maior parte destes estudos referese às populações arbóreas de florestas temperadas que, como é sabido, têm uma dinâmica diversa da floresta tropical.

Estudos sobre a estrutura genética espacial das espécies arbóreas tropicais ainda são poucos, tendo como exemplos os trabalhos de Dewey \& Heywood (1988) com Psychotria nervosa (Rubiaceae), Doligez \& Joly (1997) com Carapa procera (Meliaceae) e Epperson \& Alvarez-Buylla (1997) com Cecropia obtsusifolia (Moraceae). No Brasil, tem-se os estudos com Cedrela fissilis (Meliaceae), Chorisia speciosa (Bombacaceae) - (Gandara 1995 e Souza 1997 apud Giudice Neto 1999) e Genipa americana (Rubiaceae) - (Sebbenn et al. 1998).

Neste trabalho é apresentada a análise da estrutura genética espacial de Machaerium villosum Vog. (jacarandá-paulista), uma espécie arbórea freqüente no Brasil meridional, principalmente na floresta semidecídua de altitude, atingindo as árvores adultas alturas de até $30 \mathrm{~m}$ (Hoehne 1941, Lorenzi 1992). Segundo a classificação de Budowski (1965, 1970), a espécie enquadra-se nos estágios finais da sucessão, podendo ser incluída, segundo Ferreti et al. (1995), no grupo das secundárias tardias.

\section{Material e métodos}

O local de estudo foi a Fazenda Campininha, situada no Distrito de Martinho Prado Junior, município de Moji-Guaçu, SP, entre os paralelos $22^{\circ} 10^{\prime} 43^{\prime \prime}$ e $22^{\circ} 18^{\prime} 19^{\prime \prime}$ 'S e entre os meridianos 47 $8^{\circ}$ ' ${ }^{\prime \prime}$ ' e $47^{\circ} 11^{\prime} 34^{\prime \prime}$ 'W.

Duas populações naturais de Machaerium villosum Vog. foram amostradas. Uma, denominada Modelo Antrópico (MA), foi amostrada na área da Estação Ecológica de Moji-Guaçu pertencente ao Instituto Florestal e a outra, denominada Modelo Natural (MN), na área da Reserva Biológica e Estação Experimen- tal de Moji-Guaçu, pertencente ao Instituto de Botânica, ambos da Secretaria do Meio Ambiente do Estado de São Paulo.

A área amostral da população MA é composta por mata ciliar que acompanha o Rio Moji-Guaçu, gradando para mata mesófila, cerrado e várzeas. Esta área esteve sujeita a cortes seletivos e retirada da vegetação para implantação de culturas e pastagens (Eiten 1963, Gibbs \& Leitão-Filho 1978). Em alguns trechos da área, a vegetação fica sobre solos de planície de inundação (hidromórficos) que estão sujeitos a alagamentos periódicos na época de cheia do rio. Nessas áreas, há baixa diversidade de espécies, provavelmente devido ao estresse provocado pela inundação, exigindo tolerância das espécies.

A área de amostragem da população MN, localizada no Setor SPNP-1 da Reserva (Struffaldi-De Vuono et al. 1982, Pinto \& Struffaldi-De Vuono 1992), é composta por variação vegetal desde cerrado até mata, encontrando-se pouco perturbada.

As populações foram delimitadas por uma faixa contígua, na qual ocorressem indivíduos de M.villosum. Na população MA, em área aproximada de 300 ha, 119 indivíduos, divididos entre adultos e jovens de $M$. villosum, foram marcados. Considerou-se adulto o indivíduo que tivesse DAP (diâmetro à altura do peito) igual ou superior a $10 \mathrm{~cm}$, pois, por meio de inspeção visual das populações, constatou-se que indivíduos com este porte eram potencialmente reprodutivos. Desta forma, nesta população, 26 indivíduos adultos $(\overline{\mathrm{DAP}}=24,14 \pm 6,25 \mathrm{~cm})$ e 93 jovens $(\overline{\mathrm{DAP}}=3,24 \pm 0,53 \mathrm{~cm}$ ) foram amostrados. Na população $\mathrm{MN}$, 31 indivíduos adultos $(\overline{\mathrm{DAP}}=23,16 \pm 3,10 \mathrm{~cm})$ foram marcados, em uma área aproximada de 3 ha. A junção entre indivíduos adultos e jovens na população MA deve-se à forma com que os indivíduos estão dispostos nesta população. Observa-se que a partir de alguns indivíduos de porte maior, forma-se um agrupamento de indivíduos mais jovens com tamanhos e diâmetros variáveis. Entre um agrupamento e outro, indivíduos adultos ocorrem de forma esparsa. Assim, a reunião de todos os indivíduos tem o objetivo de captar possíveis estruturações dentro dos agrupamentos.

Sokal \& Oden (1978b) dão um exemplo de união de diferentes estágios de vida em uma mesma análise, não sendo prejudicadas as conclusões, antes o contrário, o padrão de distribuição dos diferentes estágios pôde explicar determinadas estruturações. Há ainda, estudos que utilizam árvores maduras (Perry \& Knowles 1991, Schnabel et al. 1991, Leonardi \& Menozzi 1996), sem especificar o estágio dos indivíduos e trabalhos que utilizam somente árvores adultas (Loiselle et al 1995, Doligez \& Joly 1997). Epperson \& Alvarez-Buylla (1997) analisam três estágios diferentes de vida (plântulas, juvenis e adultos) de uma espécie arbórea pioneira tropical, porém, o fazem separadamente. Ressalta-se, contudo, que a reunião feita na população MA engloba indivíduos estabelecidos na população, assemelhando-se, portanto, aos estudos com indivíduos maduros. Na população $\mathrm{MN}$, não há a ocorrência de agrupamentos.

Para a localização dos indivíduos dentro das populações, utilizou-se equipamento de DGPS (Differential Global Positioning System), que consiste de um receptor móvel para medições em campo e de um receptor fixo, por meio do qual se faz correções nas posições tomadas em coordenadas geográficas de latitude e longitude, utilizando-se o sistema global WGS 84, com Datum UGGI-79. As medidas são convertidas, posteriormente, para o sistema de coordenadas UTM (Universal Transverse Mercator), fuso 23, com o Datum SAD-69. Essa conversão permitiu a entrada 
dos dados de posicionamento de todos os indivíduos em um sistema CAD (Computer Aided Design). Desta forma, os indivíduos foram mapeados dentro de um mesmo sistema de projeção.

Amostras foliares dos indivíduos em cada população foram colhidas em agosto de 1997 e submetidas às técnicas de eletroforese de isoenzimas, segundo as recomendações descritas em Brewbaker et al. (1968), Soltis \& Soltis (1989), Kephart (1990) e Alfenas et al. (1991). A eletroforese de isoenzimas foi a horizontal, conduzida em gel de amido (penetrose 30 ) a 13\%, usando como sistema tampão Tris-citrato (TC) $\mathrm{pH} 7,5$, conforme Alfenas et al. (1991). Aproximadamente $20 \mathrm{mg}$ de tecido de limbo foliar foram retirados e macerados com um bastão de vidro em placas de porcelana, sobre barras de gelo. Às placas de porcelana, foram adicionados $10 \mathrm{mg}$ de areia lavada, $7 \mathrm{mg}$ de polivinil pirrolidona (PVP 40) e $7 \mathrm{mg}$ de PVP 360, com o objetivo de facilitar a maceração. Duas a três gotas, cerca de $300 \mu \mathrm{L}$, do extrator $\mathrm{pH} 7,3$ composto por Trizma base $0,07 \mathrm{M}$ e cisteína $0,01 \mathrm{M}$, foram colocadas e a solução final, contendo a enzima, absorvida em papel de filtro Whatman n- 3 com dimensão de $6 \times 10 \mathrm{~mm}$ (wicks). $\mathrm{O}$ gel foi cortado longitudinalmente a cerca de $2 \mathrm{~cm}$ de uma das bases para a colocação dos wicks. Nas extremidades do gel, um wick colorido com a solução azul de bromofenol a $0,1 \%$ foi colocado com a finalidade de marcar a distância máxima de migração das enzimas durante o processo eletroforético. A corrida eletroforética foi realizada em geladeira a cerca de $5{ }^{\circ} \mathrm{C}$, adicionando-se, sobre o gel, placas de vidro e cubas com gelo para evitar o superaquecimento provocado pelo atrito. A corrente elétrica permaneceu entre 32 e $35 \mathrm{~mA}$ nos primeiros $30 \mathrm{~min}$ de corrida, sendo depois elevada a $40 \mathrm{~mA}$ até o final do processo. $\mathrm{O}$ tempo médio de corrida foi de $8 \mathrm{~h}$, momento em que o marcador de azul de bromofenol atingiu a migração de 8,0 a $8,5 \mathrm{~cm}$. Após um levantamento preliminar sobre a resolução das enzimas, seis sistemas enzimáticos foram utilizados (tabela 1).

A interpretação genética dos padrões de bandeamento enzimático nos géis foi realizada seguindo padrões descritos previamente (Weeden 1983, Soltis \& Soltis 1989, Alfenas et al. 1991), identificando as zonas codificadoras dos locos e dos alelos a partir da região mais catódica para a anódica. Dez locos polimórficos foram analisados.

A análise de autocorrelação espacial foi realizada para os locos polimórficos de cada população, segundo a abordagem descrita em Heywood (1991). Para cada alelo, os indivíduos foram caracterizados geneticamente por frequiências alélicas de $0,0,5$ ou 1 , conforme tivessem, respectivamente, nenhuma, uma ou duas cópias do alelo em seus genótipos. Apenas os dois alelos mais freqüentes em cada loco foram considerados na análise, exceto quando o loco foi dialélico e, portanto, apenas um deles foi analisado, pois o outro tinha freqüência dependente.

Os métodos de conexão dos pares de localidades das variáveis empregados foram a conexão de Gabriel, o vizinho mais próximo e as classes de distância pré-determinadas (Sokal \& Oden 1978a, Dewey \& Heywood 1988, Heywood 1991). A conexão de Gabriel, a qual conecta dois pares de pontos A e B, se nenhum indivíduo ocorrer sobre ou dentro de um círculo cujo diâmetro é o segmento de reta AB (Sokal \& Oden 1978a), tem a vantagem de gerar mais pares significativos do que o método do vizinho mais próximo, reduzindo assim o erro padrão das estimativas das estatísticas de autocorrelação e como desvantagem, o método aumenta a distância média entre locais pareados (Heywood 1991).
Segundo este autor, o método do vizinho mais próximo deve prover as maiores autocorrelações, porque o parentesco entre os indivíduos é esperado diminuir com a distância, ou seja, indivíduos vizinhos seriam mais correlacionados que indivíduos mais distantes. O método de classes de distância possibilitou a construção dos correlogramas alélicos.

O coeficiente de autocorrelação empregado na análise foi o índice I de Moran (Sokal \& Oden 1978a). Segundo estes autores, o I-Moran varia de -1 (autocorrelação negativa completa, ou seja, os indivíduos pareados são completamente diferentes geneticamente) a +1 (autocorrelação positiva completa, ou seja, os indivíduos pareados são idênticos), com esperança matemática aproximando-se de zero na ausência de autocorrelação para tamanhos amostrais grandes.

Foram utilizados os programas de computador "Autocorr" desenvolvido por J.S. Heywood do Departamento de Biologia da Universidade do Estado de Missouri (EUA) e o "SAAP - Spatial Autocorrelation Analysis Program", versão 4.3, desenvolvido por D. Wartenberg da Universidade de Medicina e Odontologia de Nova Jersey (EUA).

O “Autocorr” estima o valor I-Moran, seu desvio padrão e o desvio padrão normal, conforme o método de conexão empregado. Conforme Sokal \& Oden (1978a), sob aleatoriedade a esperança de I é próxima a zero com a distribuição dos desvios padrões assintoticamente gausseana. Assim, a significância de I foi obtida pelo desvio padrão normal, sendo significativo aos níveis de probabilidade de 0,05 e 0,01 , sempre que excedesse a 1,96 ou 2,58 , respectivamente.

No método de classes de distâncias, o número de classes foi determinado em função do número de conexões em cada classe, estabelecendo-se um número mínimo de 30 conexões por classe. Na população MA, 10 classes de distância foram executadas: 1) $0-5 \mathrm{~m}$; 2) $5-10 \mathrm{~m}$; 3) $10-20 \mathrm{~m}$; 4) $20-30 \mathrm{~m}$; 5) $30-50 \mathrm{~m}$; 6) $50-150 \mathrm{~m}$; 7) $150-1000 \mathrm{~m}$; 8) $1000-2500 \mathrm{~m}$; 9) $2500-3000 \mathrm{me}$ 10) $3000-3200 \mathrm{~m}$. Na população $\mathrm{MN}$, cinco classes foram analisadas, exceto para os alelos dos locos enzimáticos 6Pgdh e Skdh, em que quatro classes puderam ser analisadas. As classes de distância desta população foram: 1) $0-50 \mathrm{~m}$; 2) $50-80 \mathrm{~m}$; 3) 80-120 m; 4) $120-170 \mathrm{~m}$; 5) 170-335 m. A classe de distância quatro dos locos 6Pgdh e Skdh corresponde ao intervalo de distância entre 120 e $335 \mathrm{~m}$.

O "SAAP" calculou o valor de I-Moran em cada classe de distância e deu a sua significância, obtida pela transformação do desvio padrão do valor observado de I-Moran em relação à sua expectativa, em desvio padrão normal. Assim, a significância indica uma autocorrelação positiva ou negativa em relação à expectativa de ausência de autocorrelação, demonstrando maior ou menor similaridade naquela classe de distância do que seria esperado pelo acaso, considerando este o único fator determinante da distribuição espacial do alelo analisado. A significância estatística dos correlogramas foi avaliada pelo método de Bonferroni segundo sugerido por Oden (1984).

\section{Resultados e Discussão}

Os valores de I-Moran calculados segundo os métodos da conexão de Gabriel e do vizinho mais 
Tabela 1. Sistemas enzimáticos e métodos de coloração utilizados em populações naturais de Machaerium villosum em Moji-Guaçu, SP.

\begin{tabular}{|c|c|c|c|}
\hline Enzima & Sigla & Referência (E.C.) & Coloração \\
\hline Alfa-esterase & $\alpha$-Est & E.C. 3.1.1.1 & $\begin{array}{l}80 \mathrm{~mL} \text { de fosfato de sódio } 0,05 \mathrm{M} \mathrm{pH} 6,0 ; 40 \mathrm{mg} \\
\text { de } \alpha \text {-naftil acetato diluído em acetona 50\%; } 30 \mathrm{mg} \\
\text { de Fast Garnet GBC diluído em n-propanol }\end{array}$ \\
\hline 6-Fosfogluconato desidrogenase & 6PGDH & E.C. 1.1.1.44 & 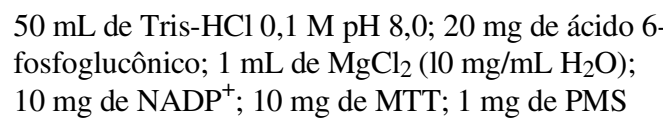 \\
\hline Fosfoglucose Isomerase & PGI & E.C.5.3.1.9 & $\begin{array}{l}50 \mathrm{~mL} \text { de Tris- } \mathrm{HCl} 0,1 \mathrm{M} \mathrm{pH} \mathrm{8,0;30} \mathrm{mg} \mathrm{de} \mathrm{frutose} \\
\text { 6-fosfato; } 1 \mathrm{~mL} \text { de } \mathrm{MgCl}_{2}\left(10 \mathrm{mg} / \mathrm{mL} \mathrm{H} \mathrm{H}_{2} \mathrm{O}\right) ; 10 \mathrm{mg} \\
\text { de } \mathrm{NADP}^{+} ; 10 \mathrm{mg} \text { de } \mathrm{MTT}, 10 \mathrm{mg} \text { de PMS, } 10 \mu \mathrm{L} \\
\text { de glucose 6-fosfato }\end{array}$ \\
\hline Malato Desidrogenase & $\mathrm{MDH}$ & E.C.1.1.1.37 & $\begin{array}{l}50 \mathrm{~mL} \text { de Tris- } \mathrm{HCl} 0,1 \mathrm{M} \mathrm{pH} \mathrm{8,5;4} \mathrm{mL} \mathrm{de} \mathrm{DL-} \\
\text { ácido málico 0,5 M pH 8,0;10 mg NAD; } 10 \mathrm{mg} \text { de } \\
\text { MTT, } 1 \mathrm{mg} \text { de PMS }\end{array}$ \\
\hline Peroxidase & PRX & E.C.1.11.1.7 & $\begin{array}{l}50 \mathrm{~mL} \text { de Acetato de sódio } 0,1 \mathrm{M} \mathrm{pH} 4,5 ; 30 \mathrm{mg} \text { de } \\
\text { 0-dionisidina bi- } \mathrm{HCl} \text { diluído em } 25 \mathrm{~mL} \text { de etanol; } \\
1 \mathrm{~mL} \text { de } \mathrm{H}_{2} \mathrm{O}_{2}(3 \%)\end{array}$ \\
\hline Xiquimato Desidrogenase & SKDH & E.C.1.1.1.25 & $\begin{array}{l}50 \mathrm{~mL} \text { de Tris- } \mathrm{HCl} 0,1 \mathrm{M} \mathrm{pH} \mathrm{8,5;60} \mathrm{mg} \mathrm{de} \mathrm{ácido} \\
\text { xiquímico; } 10 \mathrm{mg} \text { de } \mathrm{NADP}^{+} ; 10 \mathrm{mg} \text { de MTT e } \\
1 \mathrm{mg} \text { de PMS }\end{array}$ \\
\hline
\end{tabular}

E.C. $=$ Enzyme Commission .

próximo para os alelos das duas populações estão na tabela 2.

Na população MA, apenas dois alelos (alelos 2 e 3 da Pgi-1) entre 15 foram positivamente significativos pela conexão de Gabriel. Para os mesmos locos e alelos desta população, não houve coeficientes de autocorrelação espacial significativos, calculados pelo método do vizinho mais próximo.

O alelo 2 do loco Mdh- 1 e os alelos 1 e 2 do loco Skdh demonstraram uma autocorrelação positiva significativa pelo método da conexão de Gabriel na população MN. Quanto ao método do vizinho mais próximo, apenas o alelo 2 do loco enzimático Prx-2 foi significativo positivamente nesta população.

De um modo geral, os índices de Moran situaram-se próximos a zero para quase todos os locos enzimáticos analisados em ambos os métodos na população MA. Na população MN, houve uma variação dos índices, tornando-se ora pouco positivos, ora pouco negativos, porém, a maioria dos alelos não demonstrou autocorrelação espacial.

Os coeficientes de autocorrelação espacial (IMoran) calculados para classes de distâncias estão demonstrados na tabela 3. Estes coeficientes permitiram a construção dos correlogramas alélicos, os quais são mostrados nas figuras 1 a 4. Para melhor visualização os correlogramas foram divididos de acordo com as suas significâncias. Assim, as figuras 1 e 3 mostram a forma dos correlogramas significativos das duas populações estudadas. As figuras $2 \mathrm{e}$ 4 mostram os correlogramas não significativos, porém, somente os com probabilidade superior a $50 \%$ $(\mathrm{P}>0,50)$ são demonstrados.

Três correlogramas na população MA e quatro na população $\mathrm{MN}$ foram significativos pelo teste de Bonferroni (tabela 3). Estes correlogramas abrigaram a maior parte dos coeficientes significativos nas classes de distância $(62,1 \%$ na população MA e $76,9 \%$ na população $\mathrm{MN})$.

Houve 19,3\% (29 entre 150) coeficientes significativos em relação ao valor esperado de I na população MA e 19,7\% (13 entre 66) na população MN, nas classes de distância. Na população MA, houve uma proporção de $26,7 \%$ para a classe de distância 1 (até $5 \mathrm{~m})$ e de $13,3 \%$ para a classe $2(5-10 \mathrm{~m})$ de índices I-Moran positivos significativos, os quais 
Tabela 2. Coeficientes de autocorrelação espacial (I-Moran) em pontos conectados pela Conexão de Gabriel (CG) e método do vizinho mais próximo (VMP) para os alelos dos locos enzimáticos de Machaerium villosum em duas populações naturais em Moji-Guaçu, SP.

\begin{tabular}{|c|c|c|c|c|c|}
\hline \multirow[b]{2}{*}{ Loco } & \multirow[b]{2}{*}{ Alelo } & \multicolumn{2}{|c|}{ População "MA" } & \multicolumn{2}{|c|}{ População "MN" } \\
\hline & & $\mathrm{I}_{\mathrm{CG}}$ & IVMP & $\mathrm{I}_{\mathrm{CG}}$ & IVMP \\
\hline$\alpha$-Est & $\begin{array}{l}2 \\
3\end{array}$ & $\begin{array}{l}0,139 \\
0,109\end{array}$ & $\begin{array}{l}0,107 \\
0,075\end{array}$ & $\begin{array}{c}0,000 \\
-0,024\end{array}$ & $\begin{array}{l}0,089 \\
0,093\end{array}$ \\
\hline Mdh-1 & 2 & 0,103 & 0,191 & $0,284^{*}$ & 0,273 \\
\hline Mdh-2 & 1 & 0,078 & 0,041 & $-0,041$ & $-0,019$ \\
\hline Mdh-3 & 2 & 0,026 & $-0,089$ & - & - \\
\hline 6Pgdh & $\begin{array}{l}2 \\
3\end{array}$ & $\begin{array}{c}0,007 \\
-0,103\end{array}$ & $\begin{array}{l}-0,044 \\
-0,231\end{array}$ & $\begin{array}{l}-0,020 \\
0,175\end{array}$ & $\begin{array}{l}-0,154 \\
0,093\end{array}$ \\
\hline Pgi-1 & $\begin{array}{l}2 \\
3\end{array}$ & $\begin{array}{l}0,317 * * \\
0,317 * *\end{array}$ & $\begin{array}{l}0,232 \\
0,123\end{array}$ & $\begin{array}{l}-0,225 \\
-0,314\end{array}$ & $\begin{array}{l}0,193 \\
0,047\end{array}$ \\
\hline Pgi-2 & 1 & 0,088 & 0,060 & 0,056 & $-0,071$ \\
\hline $\operatorname{Prx}-1$ & $\begin{array}{l}2 \\
3\end{array}$ & $\begin{array}{l}0,049 \\
0,076\end{array}$ & $\begin{array}{l}0,041 \\
0,088\end{array}$ & $\begin{array}{l}-0,163 \\
-0,201\end{array}$ & $\begin{array}{l}-0,380 \\
-0,452\end{array}$ \\
\hline Prx-2 & 2 & 0,026 & 0,016 & 0,240 & $0,500 *$ \\
\hline Skdh & $\begin{array}{l}1 \\
2 \\
3 \\
\end{array}$ & $\begin{array}{c}- \\
-0,087 \\
0,078 \\
\end{array}$ & $\begin{array}{c}- \\
-0,024 \\
0,067 \\
\end{array}$ & $\begin{array}{c}0,408 * \\
0,500 * *\end{array}$ & $\begin{array}{l}0,311 \\
0,229\end{array}$ \\
\hline $\begin{array}{l}\text { No. méc } \\
\text { Distânci }\end{array}$ & $\begin{array}{l}\text { exões } \\
\text { n) }\end{array}$ & $\begin{array}{c}131 \pm 15 \\
41,35 \pm 4,27\end{array}$ & $\begin{array}{c}62 \pm 7 \\
16,96 \pm 2,66 \\
\end{array}$ & $\begin{array}{c}33 \pm 4 \\
35,00 \pm 1,97\end{array}$ & $\begin{array}{c}17 \pm 1 \\
29,07 \pm 2,70 \\
\end{array}$ \\
\hline
\end{tabular}

$* \mathrm{P}<0,05 ; * * \mathrm{P}<0,01$. Os intervalos de confiança das médias foram calculados com $95 \%$ de probabilidade.

indicam uma maior dependência entre os alelos próximos. A partir da classe 3 desta população (> $10 \mathrm{~m})$, os coeficientes de autocorrelação oscilaram entre os positivos e negativos, sendo que após $2500 \mathrm{~m}$ de distância (classe 9), os coeficientes de autocorrelação significativos, tornaram-se todos negativos, demonstrando uma maior independência entre os alelos.

Na classe de distância 1 (até $50 \mathrm{~m}$ ) na população $\mathrm{MN}$, houve uma proporção de 35,7\% dos índices I-Moran positivos significativos. Nas classes superiores, esta proporção mantém-se na média de $20 \%$ (21,4\% na classe 2 e 20,8\% entre as classes 4 e 5), porém, com as significâncias tendo predominância negativa. Ainda, na classe de distância 3 (80-120 m), nenhum caso de autocorrelação significativa foi constatado.

De um modo geral, o comportamento espacial dos alelos nas populações estudadas, é aleatório. Corrobora para isto, a grande maioria de coeficientes de autocorrelação espacial não significativos apurados pela conexão de Gabriel, vizinho mais próximo e classes de distância. A significância de 2/15 e 3/14 dos coeficientes de autocorrelação espacial calculados segundo a conexão de Gabriel para as populações MA e MN, respectivamente, e de 1/14 pelo vizinho mais próximo em $\mathrm{MN}$, estão próximas às classes de distância.

A observação dos correlogramas não significativos (figura 2 e 4), mostra uma clara tendência de disposição aleatória dos genótipos nas populações. Quanto aos correlogramas significativos (figura 1 e 3), nota-se uma tendência de estruturação em classes menores que diminui a medida que a distância aumenta, passando a haver então, autocorrelações negativas. Esse comportamento não pode ser atribuído ao isolamento pela distância.

Diferenças de comportamento entre os locos têm sido usadas para sugerir seleção natural, pois em locos seletivamente neutros, todos os genes devem 
Tabela 3. Coeficientes de autocorrelação espacial (I-Moran) para 10 classes de distância na população MA e 5 classes de distância na população MN de Machaerium villosum em Moji-Guaçu, S

\begin{tabular}{|c|c|c|c|c|c|c|c|c|c|c|c|c|c|}
\hline \multirow[b]{2}{*}{ População } & \multirow[b]{2}{*}{ Loco } & \multirow[b]{2}{*}{ Alelo } & \multicolumn{10}{|c|}{ Classes de distância $^{1}$} & \multirow[b]{2}{*}{$\begin{array}{l}\text { Significância do } \\
\text { correlograma p }\end{array}$} \\
\hline & & & 1 & 2 & 3 & 4 & 5 & 6 & 7 & 8 & 9 & 10 & \\
\hline \multirow[t]{15}{*}{ MA } & $\alpha$-Est & 2 & 0,07 & $-0,05$ & $-0,05^{*}$ & $0,05^{*}$ & $-0,06$ & $-0,01$ & $-0,01$ & 0,01 & $-0,02$ & 0,01 & $0,455^{\mathrm{ns}}$ \\
\hline & & 3 & 0,07 & $-0,03$ & $-0,05^{*}$ & 0,02 & $-0,02$ & $-0,02$ & 0,00 & $-0,01$ & $-0,02$ & 0,02 & $0,454^{\mathrm{ns}}$ \\
\hline & Mdh-1 & 2 & 0,01 & 0,00 & $-0,03$ & $-0,01$ & $-0,03$ & 0,00 & $-0,01$ & 0,00 & 0,00 & 0,00 & $1,000^{\mathrm{ns}}$ \\
\hline & Mdh-2 & 1 & 0,01 & $0,09 * *$ & $0,07 * *$ & $0,04 *$ & 0,05 & $-0,17 * *$ & $-0,07$ & $0,05 *$ & $-012 * *$ & $-0,14^{* *}$ & $0,000 * *$ \\
\hline & Mdh-3 & 2 & 0,03 & $-0,03$ & $-0,02$ & 0,01 & 0,02 & $-0,01$ & 0,05 & $-0,02$ & $-0,06$ & 0,00 & $0,782^{\mathrm{ns}}$ \\
\hline & 6Pgdh & 2 & $-0,03$ & 0,04 & $-0,04$ & 0,01 & $-0,01$ & 0,00 & 0,08 & $-0,01$ & $-0,05$ & $-0,03$ & $0,719^{\mathrm{ns}}$ \\
\hline & & 3 & $-0,06$ & $-0,03$ & $-0,03$ & 0,01 & 0,03 & $-0,01$ & 0,02 & $-0,01$ & 0,01 & $-0,01$ & $1,000^{\mathrm{ns}}$ \\
\hline & Pgi-1 & 2 & $0,25^{*}$ & 0,06 & $-0,09$ & $-0,16^{*}$ & 0,12 & $-0,08$ & 0,07 & $-0,01$ & $-0,02$ & 0,00 & $0,129^{\mathrm{ns}}$ \\
\hline & & 3 & $0,23 *$ & 0,12 & $-0,11^{*}$ & $-0,21 * *$ & $0,20 *$ & $-0,07$ & 0,03 & 0,00 & 0,07 & $-0,06$ & $0,021^{*}$ \\
\hline & Pgi-2 & 1 & $0,13 * *$ & $0,08 *$ & 0,00 & $0,09 * *$ & 0,06 & $-0,14 * *$ & 0,02 & $-0,15^{* *}$ & $-0,11 * *$ & $-0,06^{*}$ & $0,000 * *$ \\
\hline & Prx-1 & 2 & $-0,02$ & $-0,03$ & $-0,01$ & 0,05 & 0,00 & $-0,11 *$ & $-0,06$ & $0,06^{*}$ & $-0,06$ & 0,02 & $0,122^{\mathrm{ns}}$ \\
\hline & & 3 & 0,01 & $-0,03$ & $-0,01$ & 0,01 & 0,01 & $-0,09 *$ & $-0,05$ & $-0,03$ & $-0,01$ & 0,01 & $0,372^{\mathrm{ns}}$ \\
\hline & Prx-2 & 2 & $-0,01$ & 0,02 & $-0,03$ & 0,01 & $-0,01$ & $-0,01$ & $-0,06$ & 0,01 & $-0,01$ & 0,01 & $1,000^{\mathrm{ns}}$ \\
\hline & Skdh & 2 & $-0,02$ & $-0,06$ & 0,00 & 0,04 & $-0,01$ & $-0,10$ & $0,28 *$ & $-0,10^{*}$ & $-0,04$ & $-0,01$ & $0,111^{\mathrm{ns}}$ \\
\hline & & 3 & $0,12 *$ & 0,03 & $-0,05$ & $-0,02$ & $-0,01$ & $-0,07$ & 0,09 & $-0,04$ & $-0,05$ & 0,03 & $0,287^{\mathrm{ns}}$ \\
\hline \multirow[t]{14}{*}{$\mathrm{MN}$} & -Est & 2 & $-0,02$ & $-0,14$ & $-0,08$ & $-0,06$ & 0,06 & & & & & & $0,623^{\mathrm{ns}}$ \\
\hline & & 3 & 0,01 & $-0,18$ & $-0,06$ & $-0,08$ & 0,07 & & & & & & $0,578^{\mathrm{ns}}$ \\
\hline & Mdh-1 & 2 & $0,18 *$ & $-0,23^{*}$ & $-0,09$ & $0,39 * *$ & $-0,41 * *$ & & & & & & $0,000 * *$ \\
\hline & Mdh-2 & 1 & $-0,10$ & $-0,02$ & $-0,07$ & 0,02 & 0,01 & & & & & & $0,254^{\mathrm{ns}}$ \\
\hline & 6Pgdh & 2 & 0,09 & $-0,05$ & 0,07 & $-0,37 * *$ & & & & & & & $0,006 * *$ \\
\hline & & 3 & $0,16^{*}$ & 0,02 & 0,05 & $-0,53 * *$ & & & & & & & $0,000 * *$ \\
\hline & Pgi-1 & 2 & $-0,07$ & $-0,02$ & $-0,14$ & 0,08 & $-0,10$ & & & & & & $0,976^{\mathrm{ns}}$ \\
\hline & & 3 & $-0,19$ & $-0,04$ & 0,01 & $-0,03$ & $-0,04$ & & & & & & $0,966^{\mathrm{ns}}$ \\
\hline & Pgi-2 & 1 & $0,13 *$ & $-0,37 * *$ & $-0,17$ & $0,49 * *$ & $-0,17$ & & & & & & $0,000 * *$ \\
\hline & Prx-1 & 2 & $-0,07$ & 0,03 & 0,02 & $-0,21$ & 0,02 & & & & & & $0,263^{\mathrm{ns}}$ \\
\hline & & 3 & $-0,08$ & 0,06 & $-0,02$ & $-0,16$ & 0,01 & & & & & & $0,607^{\mathrm{ns}}$ \\
\hline & Prx-2 & 2 & $0,13^{*}$ & 0,06 & $-0,16$ & $-0,11$ & $-0,13$ & & & & & & $0,090^{\mathrm{ns}}$ \\
\hline & Skdh & 1 & $0,26 *$ & $-0,32 *$ & $-0,12$ & 0,00 & & & & & & & $0,064^{\mathrm{ns}}$ \\
\hline & & 2 & 0,13 & $-0,24$ & 0,01 & $-0,10$ & & & & & & & $0,331^{\mathrm{ns}}$ \\
\hline
\end{tabular}

$* \mathrm{p}<0,05, * * \mathrm{p}<0,01 . \mathrm{p}=$ Probabilidade. ${ }^{1}$ Classes de distância em metros : Pop. MA (1, 0-5; 2, 5-10; 3, 10-20; 4, 20-30; 5, 30-50; 6, 50-150; 7, 150-1000; 8, 1000-2500; 9, 2500-3000; 10, 3000-3200). Pop. MN (1, 0-50; 2, 50-80; 3, 80-120; 4, 120-170; 5, 170-335). A classe de distância 4 nos locos 6Pgdh e Skdh da Pop. MN corresponde ao intervalo 120-335 m. 


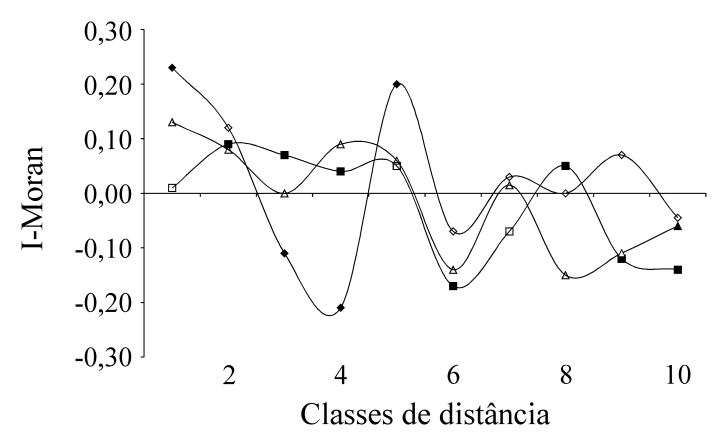

Figura 1. Correlogramas significativos dos coeficientes de autocorrelação espacial (I-Moran) em 10 classes de distância para os alelos da população MA de Machaerium villosum em MojiGuaçu, SP. Os pontos cheios indicam os coeficientes de autocorrelação significativos ao nível de $5 \%$ de probabilidade. $(\square)=$ alelo 1 Mdh-2; $(\bullet)$ alelo 3 Pgi-1; $(\Delta)=$ alelo 1 Pgi-2.

desenvolver padrões espaciais similares (Sokal \& Wartenberg 1983, Epperson \& Allard 1989). De forma igual, Sokal \& Menozzi (1982) ponderam que quando a estrutura espacial está afetando muitos locos pode ser razoavelmente interpretado como evidência de migração ou processos relacionados ao fluxo gênico, ao passo que correlogramas significativos para poucas características genéticas, podem ser seguramente interpretadas como o resultado de deriva ou seleção.

Os estudos sobre a estrutura genética espacial de populações de plantas têm revelado padrões diferenciados. Há os que obtém estruturação moderada em faixas curtas de distância e atribuem tal fato à dispersão limitada de pólen e sementes, à reprodução vegetativa e à seleção variando espacialmente (Perry \& Knowles 1991, Schnabel et al. 1991, Loiselle et al. 1995, Shapcott 1995). Há estudos que não encontraram evidências de estruturação (Leonardi \& Menozzi 1996, Doligez \& Joly 1997).

Dewey \& Heywood (1988) obtiveram estruturação significativa para um alelo entre cinco estudados, pela conexão de Gabriel para a espécie Psychotria nervosa. Suas conclusões, foram que se a estrutura para o alelo significativo for real, ela é muito fraca e existe apenas em uma escala muito local. Assim, conclui-se pela falta de estruturação na população estudada. Resultado similar foi obtido por Sebbenn et al. (1998) com Genipa americana, os quais dos cinco alelos estudados em duas populações da espécie, um foi significativo positivamente pelo

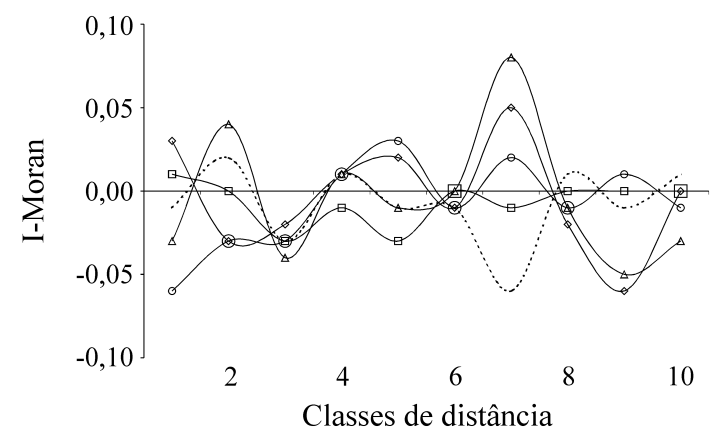

Figura 2. Correlogramas não significativos dos coeficientes de autocorrelação espacial (I-Moran) em 10 classes de distância para os alelos da população MA de Machaerium villosum em MojiGuaçu, SP. $(\square)=$ alelo $2 \mathrm{Mdh}-1 ;(\bullet)=$ alelo $2 \mathrm{Mdh}-3 ;(\Delta)=$ alelo 2 6Pgdh; $(\mathrm{O})=$ alelo 3 6Pgdh; (- - ) = alelo 2 Prx-2.

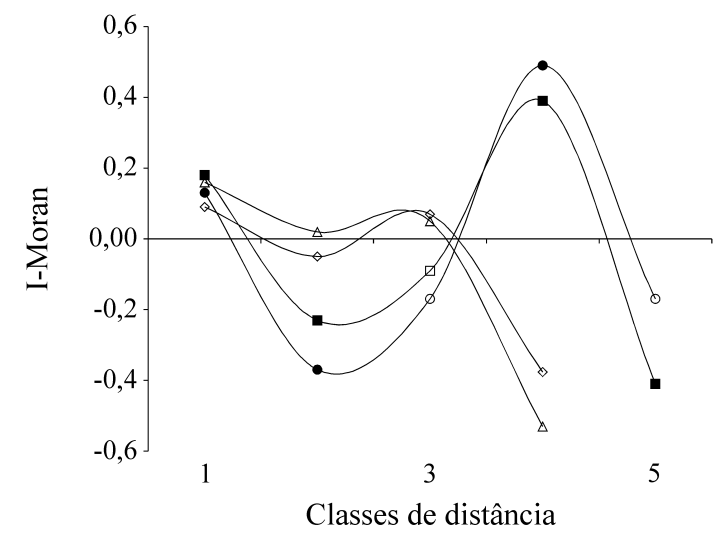

Figura 3. Correlogramas significativos dos coeficientes de autocorrelação espacial (I-Moran) em cinco classes de distância para os alelos da população MN de Machaerium villosum em MojiGuaçu, SP. Os pontos cheios indicam os coeficientes de autocorrelação significativos ao nível de $5 \%$ de probabilidade. $(\square)=$ alelo $2 \mathrm{Mdh}-1 ;(\bullet)=$ alelo 2 6Pgdh; $(\Delta)=$ alelo $36 \mathrm{Pgdh} ;(\mathrm{O})=$ alelo 1 Pgi-2.

método do vizinho mais próximo. Estes autores concluíram que não há estruturação espacial nas populações da espécie estudada, ou seja, os genótipos distribuem-se aleatoriamente nas populações.

Os resultados apresentados para o Machaerium villosum, assemelham-se de certa forma aos obtidos por Dewey \& Heywood (1988) e Sebbenn et al. (1998) e são consistentes com o padrão de dispersão de sementes pelo vento, como é esta espécie (Mantovani \& Martins 1993), que possibilita o alcance de longas distâncias, a alta taxa de cruzamento e o 


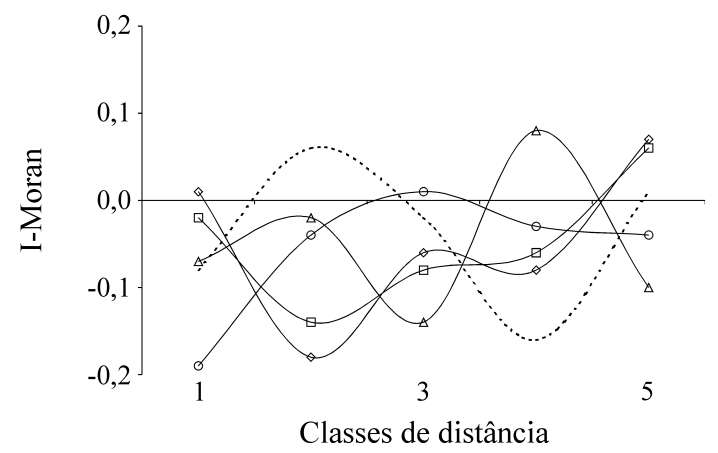

Figura 4. Correlogramas não significativos dos coeficientes de autocorrelação espacial (I-Moran) em cinco classes de distância para os alelos da população MN de Machaerium villosum em Moji-Guaçu, SP. $(\square)=$ alelo $2 \alpha$-est; $(\bullet)=$ alelo $3 \alpha$-est; $(\Delta)=$ alelo 2 Pgi-1; (O) = alelo 3 Pgi-1; (- -) = alelo 3 Prx-1.

extensivo fluxo gênico das populações analisadas (J. del Giudice Neto, dados não publicados).

Os valores significativos dos índices de Moran obtidos nos três métodos utilizados neste estudo estão próximos ou inferiores aos trabalhos com padrões de resultados similares. Além disso, a significância média de 19,5\% apurada nos correlogramas é dividida igualmente entre positiva e negativa, ou seja, reforçando ainda mais um padrão aleatório de distribuição dos genótipos.

A estruturação detectada no alelo 1 do loco Mdh-2 na população MA e no alelo 2 do loco Mdh-1 na população $\mathrm{MN}$, pode ser atribuída à alta freqüência destes alelos nas populações $(0,95$ e 0,85 , respectivamente). Epperson (1995) notou que quando a frequiência do alelo alternativo é próxima a 0,1 , é afetada a probabilidade de agrupamentos de indivíduos idênticos por descendência encontrarem e se combinar com outros agrupamentos, a medida que alguns deles espalham-se espacialmente. Leonardi \& Menozzi (1996), concluíram de forma semelhante, que locos com baixa variabilidade não devem ser incluídos na análise. Doligez \& Joly (1997), também não utilizaram alelos com freqüência superior a 0,9 ou inferior a 0,1 em suas análises. Em resumo, um alelo com alta freqüência tem menor probabilidade de se combinar com outros alelomorfos e consequentemente isto leva à estruturação espacial. Há que se notar entretanto, que outros alelos analisados também tiveram alta freqüência (J. del Giudice Neto, dados não publicados) e mesmo assim não apresentaram estruturação significativa.
Há algumas concordâncias quanto aos alelos estruturados em ambas as populações. O alelo 3 da Pgi-1 na população MA demostrou correlação significativa tanto pela conexão de Gabriel quanto nas classes de distância. Resultado similar foi verificado no alelo 2 da Mdh-1 em MN.

Nota-se contudo, que a maior parte da significância positiva para ambas as populações está na classe de distância 1. Em distâncias maiores, as autocorrelações significativas são majoritariamente negativas, isto é, alelos próximos são totalmente não relacionados. Isto explica por exemplo, a significância dos correlogramas dos alelos 2 e 3 da 6 Pgdh em $\mathrm{MN}$, em que houve dois coeficientes altamente significativos, porém negativos, na última classe de distância analisada, e que contribuíram muito para a significância do correlograma. Esses padrões são vistos para outros alelos.

A estruturação de alelos em curtas distâncias para apenas alguns locos, pode estar associada aos efeitos de seleção. Não há diferenças ambientais aparentes dentro das populações, mas pode-se notar algumas divergências entre elas. Na população MA os indivíduos encontram-se em agrupamentos. Isto pode explicar a descontinuidade de estruturação nesta população, ou seja, os pontos significativos devem estar mais intimamente relacionados dentro dos agrupados. Os coeficientes significativos demonstram a extensão do agrupado na população MA, o qual vai até a classe $6(\leq 50 \mathrm{~m})$.

Na população MN, os indivíduos estão dispostos regularmente na área, porém, não há um padrão para os pontos significativos. Grande parte desta população não encontra-se estruturada.

A atribuição à seleção para explicar a estruturação em curtas distâncias, não está em acordo com dados sobre a estrutura genética destas populações (J. del Giudice Neto, dados não publicados), sendo apresentadas evidências de seleção apenas para o loco $\alpha$-Est e não para os apontados neste estudo. Pode ser que a estrutura genética média não detectou evidências de seleção, porém, ela ocorria em escalas mais refinadas.

A baixa estruturação global das populações, levam-nos a concluir que os genótipos distribuem-se de forma aleatória dentro das populações. 


\section{Referências bibliográficas}

ALFENAS, A.C., PETERS, I., BRUNE, V. \& PASSADOR, G.C. 1991. Eletroforese de proteínas e isoenzimas de fungos e essências florestais. UFV, Viçosa.

BREWBAKER, J.L., UPADHYA, M.D., MÄKINEN, Y. \& MACDONALD, T. 1968. Isoenzyme polymorphism in flowering plants. III. Gel eletrophoretic methods and applications. Physiologia Plantarum 21:930-940.

BUDOWSKI, G. 1965. Distribution of tropical American rain forest species in the light of successional processes. Turrialba 15:4042.

BUDOWSKI, G. 1970. The distinction between old secondary and climax species in tropical central American lowland forests. Tropical Ecology 11:44-48.

DEWEY, S.E. \& HEYWOOD, J.S. 1988. Spatial genetic structure in a population of Psychotria nervosa. Distribution of genotypes. Evolution 42:834-838.

DOLIGEZ, A. \& JOLY, H.I. 1997. Genetic diversity and spatial structure within a natural stand of a tropical forest tree species, Carapa procera (Meliaceae), in French Guiana. Heredity 79:72-82.

EITEN, G. 1963. Habitat flora of Fazenda Campininha, São Paulo, Brazil. In: Simpósio sobre o cerrado (M.G.Ferri, coord.). Edgard Blücher e EDUSP, São Paulo, p.157-202.

EPPERSON, B.K. 1989. Spatial patterns of genetic variation within plant populations. In: Plant Population Genetics, breeding and genetic resource (A.H.D. Brown, M.T. Clegg, A.L. Kahler \& B.S. Weir, eds.) Sinauer Associates, Massachusetts, p.229253.

EPPERSON, B.K. 1995. Spatial distributions of genotypes under isolation by distance. Genetics 140:1431-1440.

EPPERSON, B.K. \& ALLARD, R.W. 1989. Spatial autocorrelation analysis of the distribution of genotypes within populations of Lodgepole Pine. Genetics 121:369-377.

EPPERSON, B.K. \& ALVAREZ-BUYLLA, E.R. 1997. Limited seed dispersal and genetic structure in life stages of Cecropia obtusifolia. Evolution 51:275-282.

FERRETI, A.R., KAGEYAMA, P.Y., ÁRBOCZ, G. de F., SANTOS, J.D. dos, BARROS, M.I.A de, LORZA, R.F. \& OLIVEIRA, C. de. 1995. Classificação das espécies arbóreas em grupos ecológicos para revegetação com nativas no Estado de São Paulo. Florestar Estatístico 3:73-77.

GIBBS, P.E. \& LEITÃO FILHO, H.F. 1978. Floristic composition of an area of gallery forest near Mogi Guaçu, state of São Paulo, SE. Brazil. Revista Brasileira de Botânica 1:151-156.

GIUDICE NETO, J.D. 1999. Estrutura genética por isoenzimas em populações naturais de Jacarandá Paulista (Machaerium villosum Vog.). Dissertação de mestrado, ESALQ/USP, Piracicaba.

HEYWOOD, J.S. 1991. Spatial analysis of genetic variation in plant populations. Annual Review of Ecology and Systematics 22:335-355.

HOEHNE, F.C. 1941. Flora Brasilica. Secretaria da Agricultura, Indústria e Comércio, São Paulo.

KEPHART, S.R. 1990. Starch gel electrophoresis of plant isozymes: a comparative analysis of techniques. American Journal of Botany 77:693-712.
LEONARDI, S. \& MENOZZI, P. 1996. Spatial structure of genetic variability in natural stands of Fagus sylvatica L.(beech) in Italy. Heredity 77:359-368.

LOISELLE, B.A., SORK, V.L., NASON, J. \& GRAHAM, C. 1995. Spatial genetic structure of a tropical understory shrub, Psychotria officinalis (Rubiaceae). American Journal of Botany 82:1420-1425.

LORENZI, H. 1992. Árvores brasileiras: manual de identificação e cultivo de plantas arbóreas nativas do Brasil. Plantarum, Nova Odessa.

MANTOVANI, W. \& MARTINS, F.R. 1993. Florística do cerrado na Reserva Biológica de Moji Guaçu, SP. Acta Botanica Brasilica 7:33-60.

ODEN, N.L. 1984. Assessing the significance of a spatial correlogram. Geographical Analysis 16:1-16.

PERRY, D.J. \& KNOWLES, P.1991. Spatial genetic structure within three sugar maple (Acer saccharum Marsh.) stands. Heredity 66:137-142.

PINTO, M.M. \& STRUFFALDI-DE VUONO, Y. 1992. O cerrado da Reserva Biológica de Moji-Guaçu: Manutenção e supervisão da pesquisa. Revista do Instituto Florestal 4:925-929.

SCHNABEL, A., LAUSHMAN, R.H. \& HAMRICK, J.L. 1991. Comparative genetic structure of two co-ocurring tree species, Maclura pomifera (Moraceae) and Gleditsia triacanthos (Leguminosae). Heredity 67:357-364.

SEBBENN, A.M., KAGEYAMA, P.Y. \& VENCOVSKY, R. 1998. Variabilidade genética, sistema reprodutivo e estrutura genética espacial em Genipa americana $\mathrm{L}$. através de marcadores isoenzimáticos. Scientia Forestalis 53:15-30.

SHAPCOTT, A. 1995. The spatial genetic structure in natural populations of the Australian temperate rainforest tree Atherosperma moschatum (Labill.) (Monimiaceae). Heredity 74:28-38.

SOKAL, R.R. \& JACQUEZ, G.M. 1991. Testing inferences about microevolutionary processes by means of spatial autocorrelation analysis. Evolution 45:152-168.

SOKAL, R.R. \& MENOZZI, P. 1982 Spatial autocorrelation of HLA frequencies in Europe support demic diffusion of early farmers. American Naturalist 119:1-17.

SOKAL, R.R. \& ODEN, N.L. 1978a. Spatial autocorrelation in biology. 1. Methodology. Biological Journal of the Linnean Society 10:199-228.

SOKAL, R.R. \& ODEN, N.L. 1978b. Spatial autocorrelation in biology. 2. Some biological implications and four applications of evolutionary and ecological interest. Biological Journal of the Linnean Society 10:229-249.

SOKAL, R.R. \& WARTENBERG, D.E. 1983. A test of spatial autocorrelation analysis using an isolation-by-distance model. Genetics 105: 219-237.

SOKAL, R.R., JACQUEZ, G.M. \& WOOTEN, M.C. 1989. Spatial autocorrelation analysis of migration and selection. Genetics 121:845-855.

SOLTIS, D.E. \& SOLTIS, P.S. (eds.). 1989. Isozymes in plant biology. Dioscorides Press, Portland, Oregon.

STRUFFALDI-DE VUONO,Y., BARBOSA, L.M. \& BATISTA, E.A. 1982. A Reserva Biológica de Moji-Guaçu. Silvicultura em São Paulo 16A:548-558.

WEEDEN, N.F. 1983. Evolution of plant isozymes. In: Isozymes in Plant Genetics and Breeding (S.D. Tanksley \& T.J. Orton, eds.) Elsevier Science Publishers, Amsterdam, p.175-205. 